\title{
Syndrome of inappropriate secretion of antidiuretic hormone after chemotherapy with vinorelbine
}

\author{
Hiroaki Kuroda $\cdot$ Masafumi Kawamura $\cdot$ Tai Hato $\cdot$ Kazunori Kamiya $\cdot$ \\ Masahiro Kawakubo · Yotaro Izumi • Masazumi Watanabe · Hirohisa Horinouchi • \\ Koichi Kobayashi $\cdot$ Mitsuo Nakayama
}

Published online: 11 January 2008

(C) Springer-Verlag 2008

Erratum to: Cancer Chemother Pharmacol

DOI 10.1007/s00280-007-0636-z

This article was published with a missing author:

Mitsuo Nakayama

Dr. Mitsuo Nakayama is Associate Professor, Division of General Thoracic Surgery, Saitama Medical University, Kamoda-tsujidomachi 1981, Kawagoe, Saitama 350-8550, Japan.

The online version of the original article can be found under doi:10.1007/s00280-007-0636-z.

H. Kuroda · M. Kawamura ( $₫) \cdot$ T. Hato · K. Kamiya ·

M. Kawakubo · Y. Izumi · M. Watanabe $\cdot$ H. Horinouchi ·

K. Kobayashi

Division of General Thoracic Surgery, School of Medicine,

Keio University, 35 Shinanomachi, Shinjuku-ku,

Tokyo 160-8582, Japan

e-mail: kawamura@sc.itc.keio.ac.jp

M. Nakayama

Division of General Thoracic Surgery,

Saitama Medical University,

Kamoda-tsujidomachi 1981, Kawagoe,

Saitama 350-8550, Japan 